\title{
Nasal Schwannoma: A Challenging Diagnosis of a Rare Neurofibromatosis Type 2 Manifestation
}

\author{
Thiago Sasso Carmona de Souza, MD*, Nicole Tássia Amadeu, MD, Luana Carolina Fontana, \\ MD, Rogério Hamerschmidt, PhD and Paulo Eduardo Przysiezny, MD \\ Department of Otolaryngology and Head and Neck Surgery - Hospital de Clínicas Complex, Federal University \\ of Paraná, Curitiba, Brazil
}

\begin{abstract}
Neurofibromatosis type 2 (NF2) is an autosomal dominant disease characterized by the growth of benign tumors in the nervous system. It has variable clinical manifestations, but nasal complaints are usually rare. In this paper, we present a case report of nasal schwannoma in a patient with NF2. The present report highlights the importance of investigating those patients with such underlying disease and complaining of nasal obstruction, especially in the presence of unilateral nasal mass.
\end{abstract}

Keywords

Neurofibromatosis 2, Neurilemmoma, Nasal obstruction

\section{Introduction}

Neurofibromatosis type 2 (NF2) is related to the growth of benign tumors in both the central and peripheral nervous systems. Its diagnosis usually occurs in the first two decades of life, but it can be delayed up to seven years, and there must always be a high clinical suspicion [1-3]. Although it presents different clinical manifestations, nasal complaints are rarely reported. Nasal schwannoma is a rare benign peripheral neural non-invasive tumor, of slow progression [4] and few reports of its association with NF2 have been described $[5,6]$. The purpose of this report is to describe a rare case of nasal schwannoma in a patient with NF2. This publication was authorized by the patient and duly approved by the institution's ethics committee (\#4.161.863).

\section{Case Presentation}

A 60-year-old female referred to the otolaryngology head and neck surgery service of a tertiary health center due to progressive nasal obstruction, purulent rhinorrhea, episodes of self-limited epistaxis and anosmia, with two years of evolution. She reported worsening of symptoms in the past two months. She had a diagnosis of NF2 in adolescence, with multiple excesses of skin tumors, including a history of a left side vestibular schwannoma (VS), resected in 2001, requiring reconstruction of the left facial nerve at the same surgical time through hypoglossal-facial anastomosis, remaining with a certain degree of facial paresis (House-Brackmann III). The anterior rhinoscopy and flexible fiberoptic nasopharyngoscopy showed an irregular mass in the right nasal cavity, grayish-white colored, associated with ipsilateral purulent secretion and obstructive septal deviation on the left. Oroscopy and otoscopy had no clinical changes. A biopsy of the nasal lesion performed previously at another service showed signs of chronic, inconclusive inflammation. A face computed tomography (CT) scan was performed, which showed mass in the right nasal cavity, laterally displacing the medial wall of the right maxillary sinus and the nasal septum. The right maxillary, frontal and ethmoid sinuses were filled with material with soft-tissue density (Figure 1). In the magnetic resonance imaging (MRI), it was possible to observe heterogeneous expansive lesion with a predominance of high signal in $\mathrm{T} 2$ sequence and low signal in T1, occupying a large part of the right nasal cavity, measuring $51 \times 37 \times 26 \mathrm{~mm}$, presenting restriction to water diffusion and intense contrast highlight, without signs of intracranial extension (Figure 2).

To proceed the investigation, we chose to perform an excisional biopsy of the nasal mass under endoscopic vision (Figure 3). The result of the histopathological analysis of the

*Corresponding author: Dr. Thiago Sasso Carmona de Souza, MD, Department of Otolaryngology and Head and Neck Surgery - Hospital de Clínicas Complex, Federal University of Paraná, R Gen Carneiro, 181 - Alto da Glória, Curitiba, Paraná, 80060-900, Brazil, Tel: +55-41-999997892

Accepted: November 03, 2020

Published online: November 05, 2020

Citation: de Souza TSC, Amadeu NT, Fontana LC, et al. (2020) Nasal Schwannoma: A Challenging Diagnosis of a Rare Neurofibromatosis Type 2 Manifestation. J Head Neck Surg 2(1):70-73 

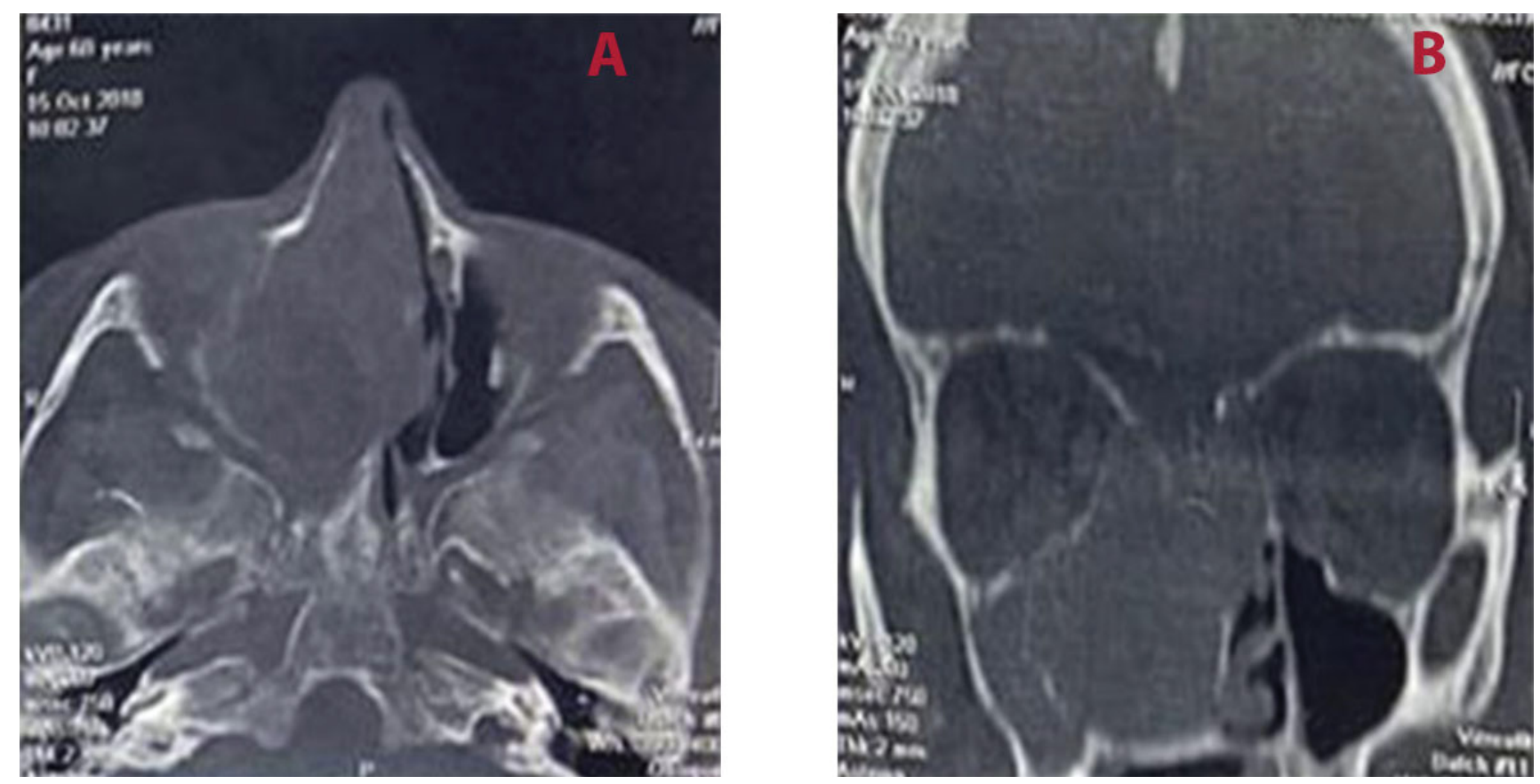

Figure 1: (A) Axial section computed tomography showing soft-tissue density material in the right nasal cavity, maxillary and ethmoid sinus; the medial displacement of the septal region and the medial wall of the maxillary sinus is observed; (B) Tomographic section in coronal view, where we can see a soft-tissue density mass.

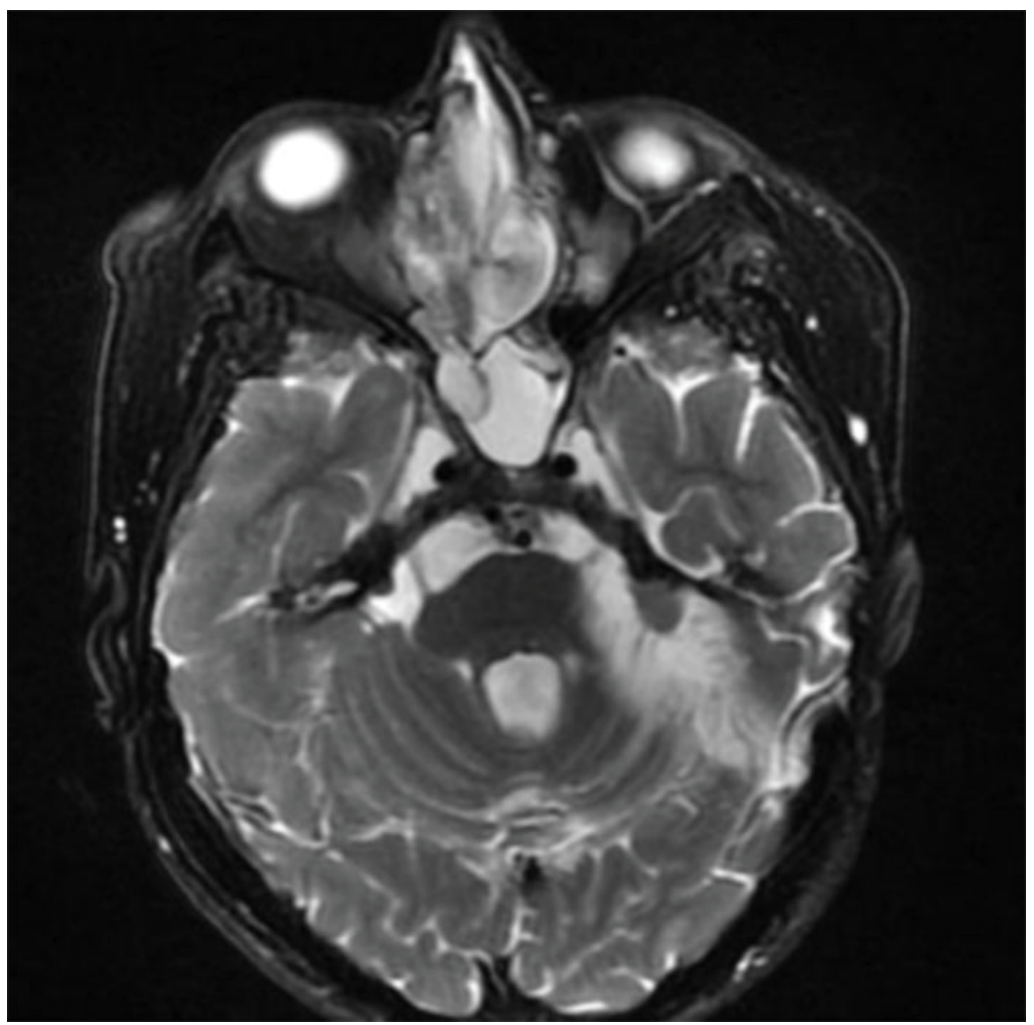

Figure 2: Magnetic resonance imaging showing heterogeneous expansive lesion with predominance of high signal in T2 sequence.

mass presented with an inconclusive atypical morphology arrangement, at first evaluation suggesting a glioma. After a detailed review and discussion, a complementation exam with immunohistochemistry was performed, confirming the diagnosis of nasal schwannoma, due to the intense immunos- taining of the S-100 protein. After resection, the patient had significant improvement of nasal symptoms, remaining completely asymptomatic and with no signs of recurrence at 12 months postoperatively. 


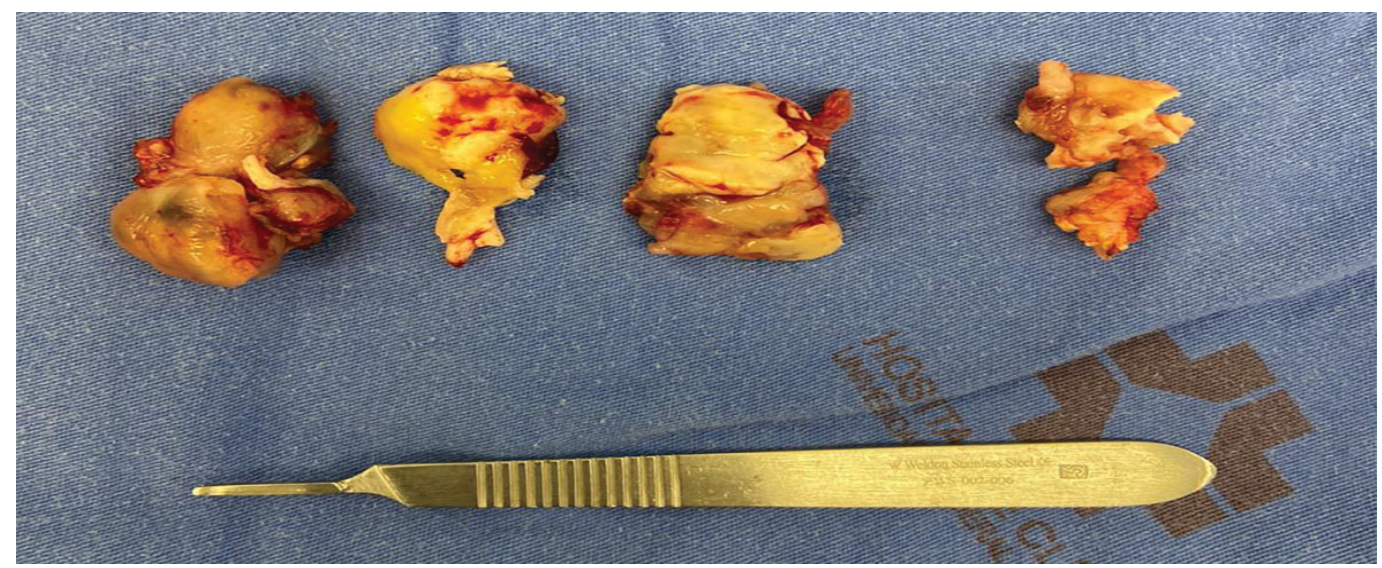

Figure 3: Excisional biopsy product of nasal mass.

\section{Discussion}

The first record of neurofibromatosis occurred in 1768, through the description of cutaneous neurofibromas. In 1882, the German physician Von Recklinghausen described the condition completely, confirming the nervous origin of the tumors [7]. Neurofibromatosis (NF) is classified into three types: Neurofibromatosis type 1 (NF1), NF2 and schwannomatosis. NF2 is the rarest, occurring in 1:25,000-40,000 live births, with an estimated prevalence of 1:60,000-200,000 individuals, since manifestations commonly begin in the second or third decade of life and some carriers do not survive in time of diagnosis $[1,8]$. It is a disease with autosomal dominant inheritance, incomplete penetrance and variable expression. It results from mutations in chromosome 22, leading to dysfunction of the tumor-suppressing merlin protein, causing the growth of benign tumors in the central and peripheral nervous system, particularly bilateral VS, present in $90-95 \%$ of patients [3]. The main symptoms are hearing loss, dizziness, imbalance and tinnitus [1].

Clinical manifestations vary in the form and age of presentation and depend on the location of the neoplasms. In addition to VS, meningiomas, astrocytomas, neurofibromas and other low-grade malignancies, such as ependymomas and gliomas can usually be found in the spinal cord [1,9]. Other presentations include peripheral neuropathy, ophthalmological manifestations and tumor skin lesions [1]. Laryngological manifestations of NF have already been described in patients with complaints of dysphonia and dysphagia, including primary laryngeal tumors. More often, unilateral or bilateral vocal fold paralysis can occur [10]. Nasal involvement of NF2, as found in the present report, is extremely rare [5].

Approximately $25-45 \%$ of schwannomas occur on the head and neck, with nasosinusal schwannomas corresponding to less than $4 \%$ of schwannomas in this region $[5,6,11]$. Less than 100 cases of nasal schwannoma (neurilemoma) have been described in the English-language literature since 1943 and its association with NF2 has been reported in isolated cases. According to the literature it is not possible to estimate its real prevalence in these patients, but it is known that the majority occurs in adults in the fourth and sixth decade of life $[5,11]$. The ethmoid sinus is the most affected, followed by the maxillary sinus, nasal cavity, including the nasal septum, and sphenoid sinus [12]. Symptoms of nasal neurilemoma are non-specific, resulting from a mass effect or tumor necrosis. Thus, if there is no high clinical suspicion, in view of the similarity with other more frequent diseases, its diagnosis may be delayed. The main differential diagnoses of unilateral nasal masses include nasal polyposis, antrochoanal polyp, chronic rhinosinusitis, bullous middle concha, mucous retention cyst, mucocele, and, more rarely, neurofibroma, inverted papilloma, malignant tumors of the nerve sheath, fibromyxoma and sarcoma [5,6].

In patients with NF2 and nasal complaints, a detailed otorhinolaryngological evaluation with flexible fiberoptic nasopharyngoscopy should be performed, in association with complementary imaging tests to better characterize the lesions [5]. The head CT scan evaluates the relationships of the lesion with the adjacent bone structures and the skull MRI is necessary to differentiate inflammatory tissue from normal tissue and to assess extension to the central nervous system. Both are required for planning surgical treatment $[5,6]$. In this presentation, there were no signs of bone erosion, orbital or intracranial invasion. The presence of bone remodeling, with displacement of the sinus wall, but without destruction, reinforces the diagnosis of a benign tumor [12].

The presence of unilateral nasal mass should ideally be investigated with biopsy, which is considered the gold standard [11]. In controversial cases, immunohistochemistry assists in the correct diagnosis $[6,12]$. In the present case, diffuse positivity of the S-100 protein was essential for the characterization and confirmation of schwannoma, differentiating it from other peripheral nerve sheath tumors [11,12].

The treatment of choice for neurilemoma is the complete excision of the tumor, since these tumors are generally resistant to radiotherapy. Functional and aesthetic complaints should be considered for surgical planning, as the tumor is non-invasive and slow-growing in nature [5]. Thus, the optimized treatment must be multidisciplinary and individualized, due to the complexity and multiplicity of injuries, which are usually progressive with advancing age [1]. In our case, we opted for the endoscopic approach, considering its lower morbidity and length of hospital stay, in addition to an 
aesthetic advantage due to the absence of a cutaneous scar when compared to an open access procedure [6].

The prognosis of tumors confined to the paranasal sinuses is excellent [6], and recurrence is rare after complete excision. However, cases of malignant transformation of schwannomas have been reported [11]. Therefore, long-term follow-up should be performed, if possible with nasal endoscopy. Our patient presented a favorable evolution and did not show signs of recurrence in one year of follow-up. The monitoring will be maintained for an indefinite period.

The otolaryngologist - head and neck surgeon may be the first doctor to be sought by patients with NF2 [9]. The literature lacks data to estimate the prevalence of nasal manifestations in these cases. Nonetheless, the presence of a nasal mass, although rare, can be one of the clinical presentations and should always be investigated, despite the patient's background. In patients already diagnosed with NF2, detailed evaluation of otorhinolaryngological complaints and monitoring is essential as part of the multidisciplinary approach, due to the possibility, although uncommon, of tumor involvement in the head and neck region.

\section{Acknowledgements}

We would like to thank the pathology department of our institution.

\section{Conflicts of Interest}

None declared.

\section{References}

1. Asthagiri AR, Parry DM, Butman JA, et al. (2009) Neurofibromatosis type 2. The Lancet 373: 1974-1986.
2. Evans DG, King AT, Bowers NL, et al. (2019) Identifying the deficiencies of current diagnostic criteria for neurofibromatosis 2 using databases of 2777 individuals with molecular testing. Genet Med 21: 1525-1533.

3. Maccollin M (1999) Neurofibromatosis 2 - Clinical Aspects. In: Friedman JM, Gutmann DH, Collin M, Riccardi VM, Phenotype, Natural History, and Pathogenesis. ( $3^{\text {rd }}$ edn), Baltimore: The Johns Hopkins University Press, 299-326.

4. Sunaryo PL, Svider PF, Husain Q, et al. (2014) Schwannomas of the sinonasal tract and anterior skull base: A systematic review of 94 cases. Am J Rhinol Allergy 28: 39-49.

5. Fung HS, Lam PW Y, Tsang TK, et al. (2008) Nasal neurilemmoma in a patient with neurofibromatosis 2 . Journal of the Hong Kong College of Radiologists 11: 122-125.

6. Pauna HF, De Carvalho GM, Guimarães AC, et al. (2013) schwannoma de septo nasal: Avaliação de massa nasal unilateral. Brazilian Journal of Otorhinolaryngology 79: 403.

7. Huson SM, Harper PS, Compston DAS (1988) Von recklinghausen neurofibromatosis: A clinical and population study in south-east wales. Brain 111: 1355-1381.

8. Evans DG (1999) Neurofibromatosis type 2: Genetic and clinical features. Ear Nose Throat J 78: 97-100.

9. Silva Miguel, Romão JD, De PA (2017) Neurofibromatose tipo II: A propósito de casos clínicos. Revista Portuguesa De Otorrinolaringologia E Cirurgia Cérvico-Facial 54: 187-191.

10. Naunheim MR, Plotkin SR, Franco RA, et al. (2016) Laryngeal manifestations of neurofibromatosis. Otolaryngol Head Neck Surg 154: 494-497.

11. Dhawle MS, Rathod SG, Bhatkule MA, et al. (2017) Sinonasal schwannoma - A case report. J Clin Diagn Res 11: 22-23.

12. Karatas A, Cebi IT, Salviz M, et al. (2016) Schwannoma of the nasal septum. Egyptian Journal of Ear, Nose, Throat And Allied Sciences17: 185-188.

DOI: $10.36959 / 605 / 542$ 